Classification

Physics Abstracts

$61.80-79.20$

\title{
Modification de la loi de Charlesby. II. Sensibilité macroscopique
}

\author{
A. Schiltz, A. Weill et P. Paniez \\ C.N.E.T., B.P. 98, Chemin du Vieux Chêne, 38243 Meylan Cedex, France
}

(Reçu le 16 janvier 1984, accepté le 15 mars 1984)

\begin{abstract}
Résumé. - Nous avons vu dans la partie I [10], les problèmes liés à l'application de la loi de Charlesby et nous avons montré qu'elle est de nature locale.

Nous montrons que pour des valeurs de la dose faibles, proches de la sensibilité macroscopique, $Q_{\text {sm }}$, il apparaît que le flux de particules n'a plus un effet uniformément dégradateur dans toute la surface.

Cela nous conduit à proposer une modification de la loi de Charlesby, modification qui permet de résoudre les problèmes soulevés en partie I.
\end{abstract}

\begin{abstract}
Part I dealt with the problems related to the application of the Charlesby's law which turned out to be a local law.

In part II, results are presented showing that for doses below macroscopic sensitivity, $Q_{\text {sm }}$, degradation due to fluence of the particles seems to be non-uniform over the entire area.

In the light of this, a modification of the Charlesby's law is proposed providing a solution to the problems considered in part I.
\end{abstract}

\section{Sensibilité pratique et sensibilité macroscopique.}

1.1 Définitions. - Dans le cas d'une résine positive, la sensibilité pratique, $Q_{\text {sp }}$, est la valeur de la dose minimale nécessaire pour enlever toute l'épaisseur de la couche irradiée.

Elle est fonction des conditions de développe- ment [5-8]. On la détermine expérimentalement en traçant la courbe $e_{\mathrm{r}} / e_{0}=f(Q)$ représentée figure 1 . C'est la grandeur qui est utilisée pratiquement dans les procédés technologiques.

Nous définissons la sensibilité macroscopique, $Q_{\mathrm{sm}}$, comme étant la valeur de la dose minimale qui provoque une variation observable de la vitesse de dissolu-

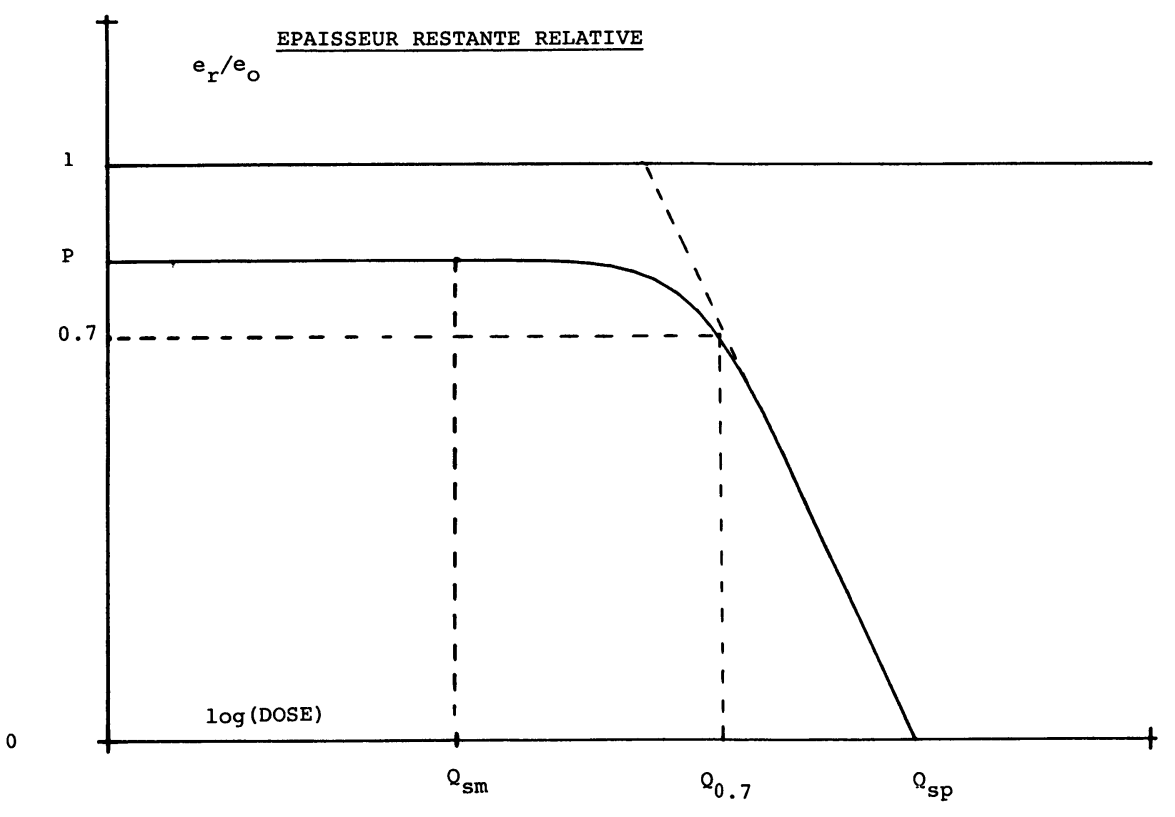

Fig. 1. - Trace de $e_{\mathrm{r}} / e_{0}=f(Q)$ dans le cas d'une résine positive.

[Schematic curve for $e_{\mathrm{r}} / e_{0}=f(Q)$ in a positive resist.] 
tion de la couche irradiée par rapport à ceile de ia couche non irradiée.

1.2 Calcul De l'épaisseur Restante, $e_{\mathrm{r}} / e_{0}$. - A partir des équations (1) et (2), on calcule la vitesse de dissolution en fonction de la dose, $V_{\mathrm{D}}(Q)$. En posant, par définition : $e_{\mathrm{r}}=\left[e_{0}-t_{\mathrm{D}} \cdot V_{\mathrm{D}}(Q)\right]$ où $t_{\mathrm{D}}$ est le temps de développement, et en supposant que l'énergie déposée, $E_{\mathrm{d}}$, est constante le long de l'épaisseur irradiée, on obtient l'expression de l'épaisseur restante relative :

$$
\begin{aligned}
\frac{e_{\mathrm{r}}}{e_{0}}=1 & -\frac{t_{\mathrm{D}}}{e_{0}} \times \\
& \times\left[A+B \cdot\left(\frac{1}{\overline{M n}, o}+\frac{G \cdot \dot{E}_{\mathrm{d}}}{100 \rho \cdot N_{\mathrm{A}}} \cdot Q\right)^{\alpha}\right] .
\end{aligned}
$$

Cette équation ne rend pas compte du phénomène de seuil qui apparaît dans les courbes expérimentales.
Sur ia Îgure 2 soni rẹprésentités les pouints cxpérimentaux décrivant la fonction $e_{\mathrm{r}} / e_{0}=f(Q)$ dans le cas électronique traité au 3.1 de la première partie. Les lignes continues représentent les courbes issues de l'équation (7) et de l'expression numérique de $1 / \overline{M n}, f=f(Q)$ obtenue au 3.1 de la première partie. Les courbes calculées avec (7) ne sont pas définies pour les valeurs de la dose inférieures à $2 \times 10^{13} \mathrm{el} . \mathrm{cm}^{2}$ et elles ne décrivent pas le phénomène de seuil tracé en pointillé.

On en déduit que la loi de Charlesby n'est pas applicable dans le domaine des faibles doses.

\section{Loi modifiée}

Nous proposons la modification suivante de la loi de Charlesby (2), représentée figure 3 :

$$
\left.\begin{array}{lr}
\frac{1}{\overline{M n}, f}=\frac{1}{\overline{M n}, o}+\frac{G \cdot E_{\mathrm{d}}}{100 \rho \cdot N_{\mathrm{A}}} \cdot\left(Q-Q_{\mathrm{sm}}\right) & \text { pour } Q>Q_{\mathrm{sm}} \\
\frac{1}{\overline{M n}, f}=\frac{1}{\overline{M n}, o} & \text { pour } Q<Q_{\mathrm{sm}}
\end{array}\right\} \text { (2-mod). }
$$

En conséquence de quoi les relations (3), (4), (6) et (7) sont modifiées en remplaçant $Q$ par $\left(Q-Q_{\mathrm{sm}}\right)$ dans (3-mod), (4-mod), (6-mod) et (7-mod).

La modification que nous proposons rend avanta- geusement compte du comportement de la résine irradiée à faible dose tout en incluant le comportement à dose élevée.

On peut maintenant appliquer la loi modifiée aux exemples précédemment traités.

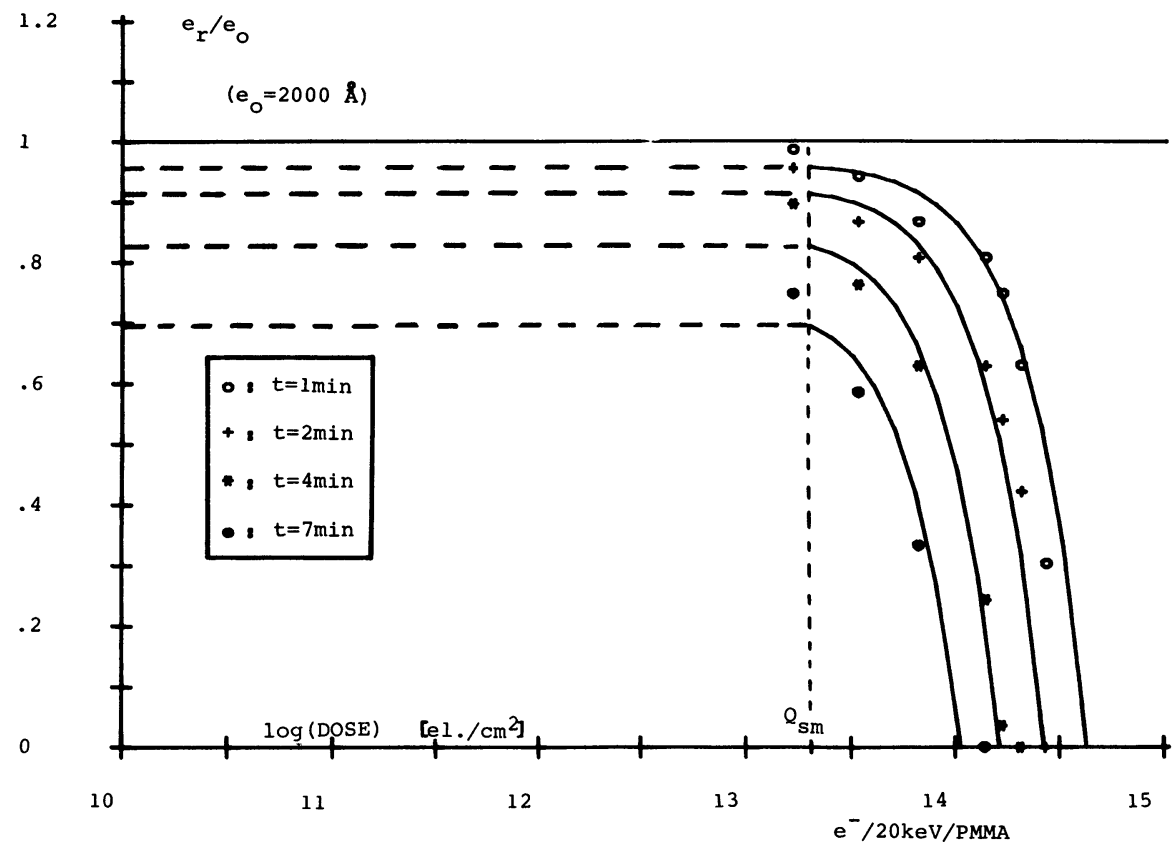

Fig. 2. - Epaisseur restante relative. Points expérimentaux et courbes théoriques.

[Normalized thickness-experimental points and theoretical curves.] 


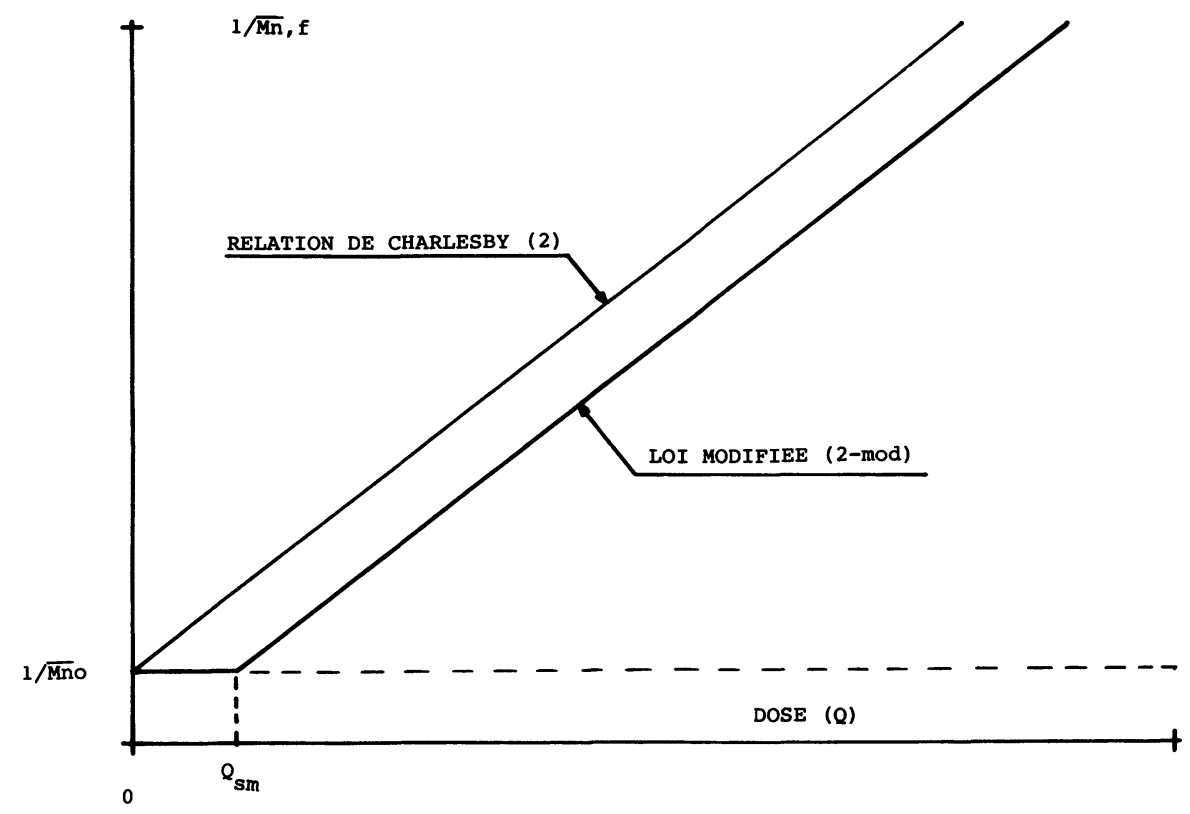

Fig. 3. - Graphes théoriques de $1 / \bar{M} n f=f(Q)$.

[Theoretical curves for $1 / \bar{M} n f=f(Q)$.]

\section{Application de la loi modifiée à deux exemples.}

3.1 Cas électronique; $\mathrm{e}^{-} / 20 \mathrm{keV} / e \ll R_{\mathrm{p}}$. Parmi les conséquences de l'application de la loi modifiée, on distingue deux résultats majeurs.

Le premier résultat concerne le calcul du taux de dégradation, $G$. Nous avons calculé le terme $\left(G . E_{\mathrm{d}}\right)$ à l'aide de l'équation (3-mod) en prenant $Q_{\mathrm{sm}}=$ $1,985 \times 10^{13} \mathrm{el} / \mathrm{cm}^{2}$. On constate figure 4 que ce terme, et par suite $G$, peuvent être assimilés à des constantes indépendantes de la dose $:\left(G \cdot E_{\mathrm{d}}\right)=5,9 \pm$ 0,5 coup. $/ 100 \mathrm{~nm}$; d'où, avec $\bar{E}_{\mathrm{d}}=2,3 \pm 0,3 \mathrm{eV} / \mathrm{nm}$ on obtient : $G=2,6 \pm 0,4$ coup. $/ 100 \mathrm{eV}$.

Le deuxième résultat concerne l'accord entre les points expérimentaux et les courbes théoriques :

- L'équation (4-mod), permet, figure 5, de relier les points expérimentaux représentant les courbes de développement $x=f\left(t_{\mathrm{D}}\right)$.

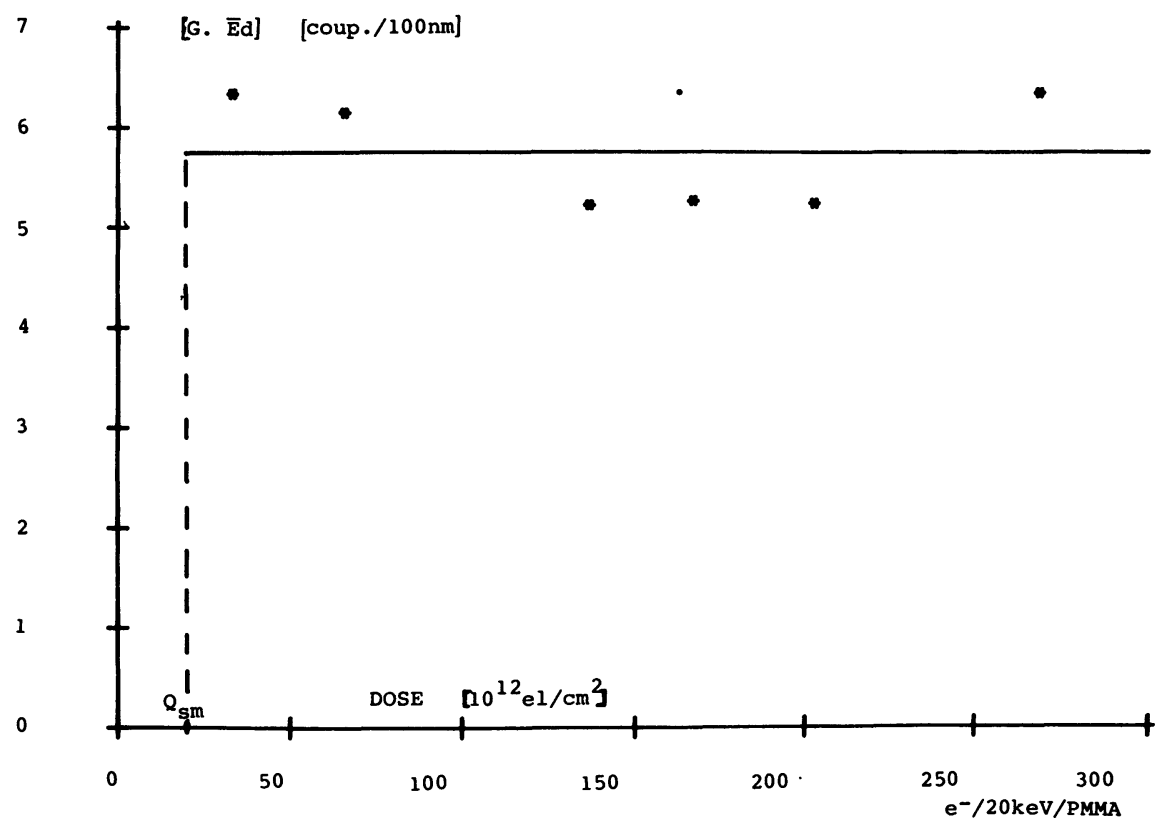

Fig. 4. - Calcul de $\left[G \cdot \bar{E}_{\mathrm{d}}\right]=f(Q)$ avec (3-mod).

[Calculated curve for $\left(G \cdot \bar{E}_{\mathrm{d}}\right)=f(Q)$ from (3-mod).] 


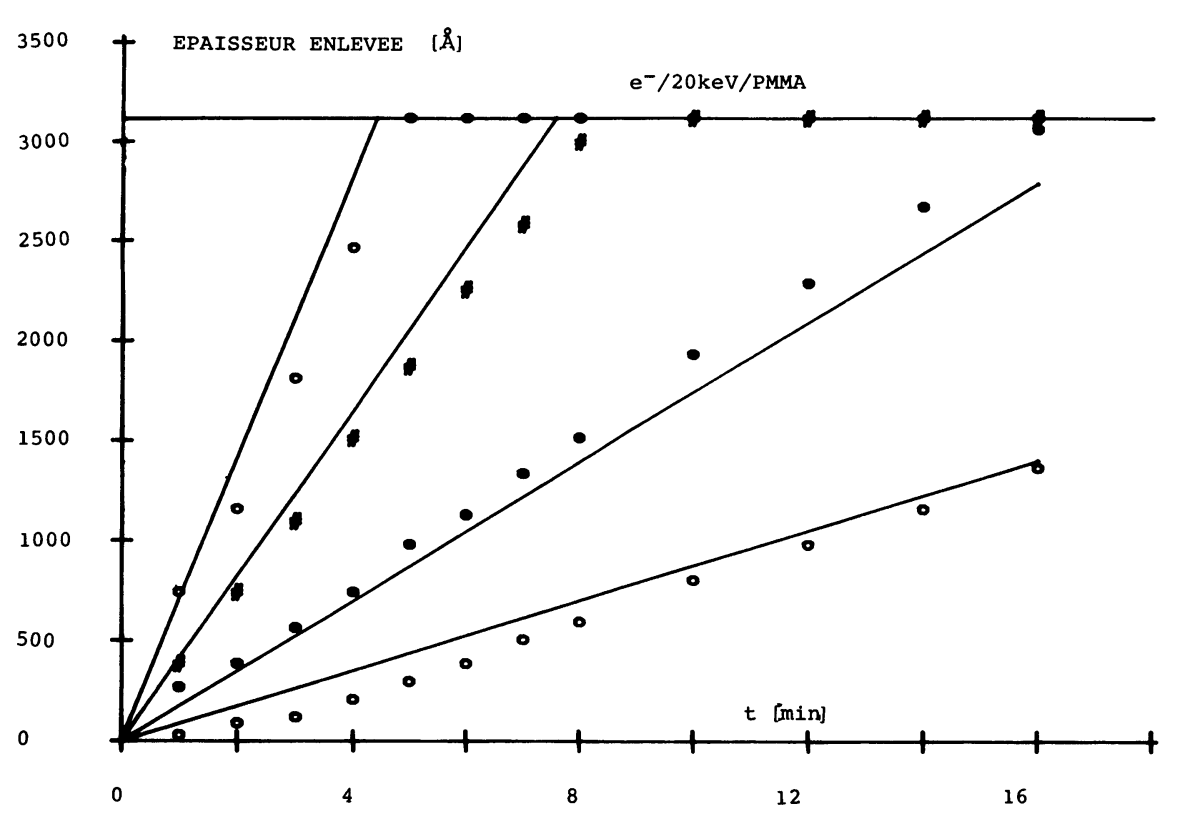

Fig. 5. - Points expérimentaux et courbes théoriques calculées avec la loi modifiée.

[Experimental points and theoretical curves calculated according to the modified law.]

- L'équation (7-mod) rend parfaitement compte du phénomène de seuil qui apparaît dans les courbes $e_{\mathrm{r}} / e_{0}=f(Q)$. En effet, les courbes obtenues se superposent à la fois aux tracés en continu et aux tracés en pointillé de la figure 2 .

3. 2 CAS IONIQUe $; \mathrm{H}^{+} / 50 \mathrm{keV} / e>R_{\mathrm{p}}$. Ce cas a été décrit au 3.2 de la première partie. L'application de la loi modifiée conduit aux résultats suivants :
- La relation (2-mod) est vérifiée pour $Q_{\mathrm{sm}}=6 \times$ $10^{12}$ ions $/ \mathrm{cm}^{2}$.

- La relation (3-mod), contrairement à la relation (3), permet d'obtenir figure 6 une courbe $\left(G \cdot E_{\mathrm{d}}\right)=$ $x$ uni ue et de vérifier que le taux de coupure est bien indépendant de la dose.

- La relation (4-mod) permet, figure 7, de relier parfaitement les points expérimentaux de la figure 3

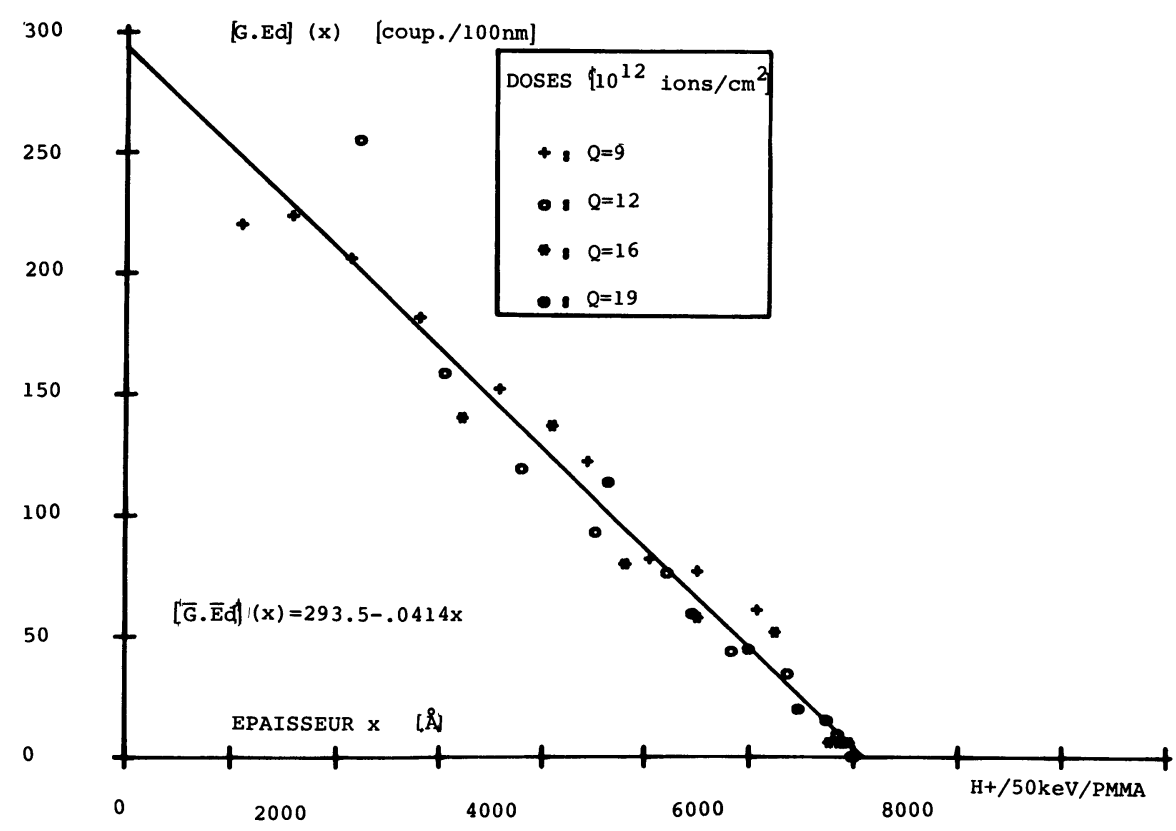

Fig. 6. - Taux $\left[G . E_{\mathrm{d}}\right](x)$ calculé avec (3-mod).

[Calculated curve for $\left(G . E_{\mathrm{d}}\right)=f(x)$ from (3-mod).] 


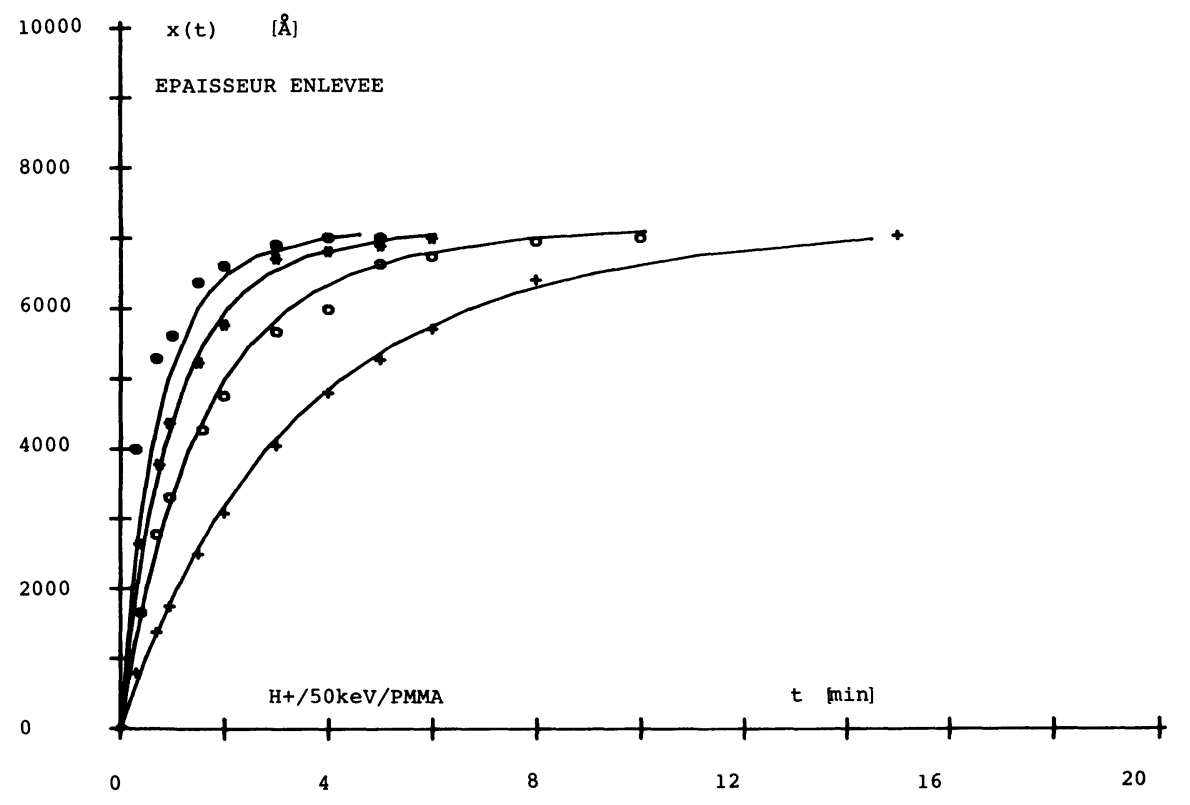

Fig. 7. - Points expérimentaux et courbes théoriques calculées avec la loi modifiée.

[Experimental points and theoretical curves calculated according to the modified law.]

de la partie I, représentant les courbes de développement $x\left(t_{\mathbf{D}}\right)$.

\section{Discussion.}

Lorsqu'une couche de résine positive est irradiée à une dose faible au point que seuls quelques picots statistiquement distribués sont irradiés, la couche ne peut pas être révélée.

On se trouve dans la partie horizontale de la figure 1.

La cinétique de dissolution de la couche de polymère irradiée n'est affectée que si la densité des picots est telle qu'ils s'interpénètrent.

Ainsi, la sensibilité macroscopique $Q_{\text {sm }}$ serait la dose d'irradiation qui crée dans le matériau une densité de picots telle que ceux-ci seraient tous tangents entre eux.

Selon cette interprétation et en admettant que les particules sont équiréparties à la surface du polymère, l'aire du picot élémentaire ainsi défini est égale à l'inverse de la valeur de $Q_{\text {sm }}$, c'est-à-dire :

$$
1 / Q_{\mathrm{sm}} \simeq 17 \mathrm{~nm}^{2} \text { dans le cas } \mathrm{H}^{+} / 50 \mathrm{keV} / \mathrm{PMMA}
$$

$$
\text { et } 1 / Q_{\mathrm{sm}} \simeq 2 \mathrm{~nm}^{2} \text { dans le cas } \mathrm{e}^{-} / 20 \mathrm{keV} / \text { PMMA. }
$$

Dans ce dernier cas, puisque l'énergie déposée est constante, le volume du picot se calcule comme : $v_{\mathrm{p}}=1 / Q_{\mathrm{sm}} \cdot G \cdot \bar{E}_{\mathrm{d}}$, soit environ $85 \mathrm{~nm}^{3}$. Le volume moyen d'une macromolécule de PMMA $(\overline{M n}, o=$ $2 \times 10^{5}$ ) étant d'environ $280 \mathrm{~nm}^{3}$, il faut donc, selon notre interprétation, en moyenne 3,3 coupures par macromolécule pour que l'effet de l'irradiation soit observable.

Il faut aussi noter que l'énergie déposée, $E_{\mathrm{d}}$, calculée à l'aide de (3-mod) et la grandeur, $R_{\mathrm{c}}$, sont toutes deux calculées à partir de la mesure, par un processus chimique, du taux de dégradation effective du polymère. Ces grandeurs sont donc par essence différentes de celles calculées à partir de théories physiques qui sont caractéristiques du comportement des particules ellesmêmes et non de leur effet sur le matériau.

Remarquons enfin que la loi proposée permet d'évaluer les deux grandeurs technologiques suivantes :

a) La sensibilité pratique, $Q_{\text {sp }}$, qui s'exprime comme :

$$
\begin{aligned}
Q_{\mathrm{sp}}=Q_{\mathrm{sm}}+ & \frac{100 \rho \cdot N_{\mathrm{A}}}{G \cdot \bar{E}_{\mathrm{d}}} \times \\
\times & {\left[\left(\frac{1}{B} \cdot\left(\frac{e_{0}}{t_{\mathrm{D}}}-A\right)\right)^{1 / \alpha}-\frac{1}{\overline{M n}, o}\right] . }
\end{aligned}
$$

Cette équation montre que la sensibilité pratique dépend, entre autres [5-8], du rapport $e_{0} / t_{\mathrm{D}}$.

b) Le contraste, $\Gamma_{0,7}$, qui se calcule comme :

$$
\Gamma_{0,7}=\left|\frac{0,7}{\log \left(Q_{0,7} / Q_{\mathrm{sp}}\right)}\right| .
$$

Il dépend lui aussi du choix du couple $\left(e_{0}, t_{\mathrm{D}}\right)$.

\section{Conclusion.}

Nous avons défini la notion de sensibilité macroscopique, $Q_{\text {sm }}$, qui est la dose minimale permettant 
d'observer une variation de la cinétique de dissolution des polymères irradiés par des particules.

En modifiant la loi de Charlesby par un décalage d'origine, de valeur $Q_{\mathrm{sm}}$, on obtient une très bonne concordance entre les résultats expérimentaux et la théorie.

Il devient ainsi possible d'évaluer des paramètres technologiques importants tels que la sensibilité pratique, $Q_{\mathrm{sp}}$, et le contraste, $\Gamma_{0,7}$.

\section{Remerciements.}

Les auteurs tiennent à remercier MM. F. Schue et J. C. Dubois pour leurs commentaires et l'intérêt qu'ils ont porté à ce travail.

\section{Notations.}

$Q_{\mathrm{sp}}:$ Sensibilité pratique (part./ $\mathrm{cm}^{2}$ ).

$Q_{\mathrm{sm}}$ : Sensibilité macroscopique (part. $/ \mathrm{cm}^{2}$ ).

$e_{0}$ : Epaisseur initiale de la couche révélée $(\AA)$.

$e_{\mathrm{r}} \quad$ : Epaisseur restante au temps de développement $t_{\mathrm{D}}(\AA)$.

$x\left(t_{\mathrm{D}}\right):$ Epaisseur enlevée au temps $t_{\mathrm{D}}: e_{\mathrm{r}}=e_{0}$ $x\left(t_{\mathrm{D}}\right)$.

$V_{0}:$ Vitesse de dissolution de la résine non irradiée :

$$
V_{0}=A+B \cdot(1 / \overline{M n}, o)^{\alpha} .
$$

$Q_{0,7}$ : Dose pour laquelle $\left(e_{\mathrm{r}} / e_{0}\right)=0,7$.

\section{Bibliographie}

[1] Dole, M., Radiation Chemistry of Macromolecules (Academic Press) 1972.

[2] Ku, H. Y. et Scala, L. C., J. Electrochem. Soc. 116 (1969) 980.

[3] Ryssel, H. et HABERGER, K.' Ion Beam Lithography, Conference ME (1981).

[4] Komuro, M., Atoda, N. et Kawakatsu, H., J. Electrochem. Soc. 126, 3 (1979) 483.

[5] Greeneich, J. S., J. Electrochem. Soc. 122 (1975) 970.

[6] Hall, T. M., Wagner, A. et Thompson, L. F., J. Appl. Phys. 53, 6 (1982) 3997.
[7] Adesida, I., Ion Bombardments of Resists (1982).

[8] Eranian, A. et Dubois, J. C., Matériaux pour lithographie 1387/81. LCR, GR2, Thomson CSF (1981).

[9] Karapiperis, L., Adesida, I., Lee, C. A. et Wolf, E. D., J. Vac. Sci. Technol. 19 (1981) 4.

[10] Schiltz, A., Weill, A. et Paniez, P., Modification de la loi de Charlesby. Partie I, Revue Phys. Appl. 19 (1984); ce numéro. 